\title{
The onset of compressibility effects for aeroiolils in ground effect
}

\author{
G. Doig, T. J. Barber and E. Leonardi \\ School of Mechanical and Manufacturing Engineering \\ University of New South Wales \\ Sydney, Australia
}

\author{
A. J. Neely \\ School of Aerospace, Civil and Mechanical Engineering \\ University of New South Wales at the Australian Defence Force Academy \\ Canberra, Australia
}

\section{ABSTRACT}

The influence of flow compressibility on a highly-cambered inverted acrofoil in ground effect is presented, based on two-dimensional computational studies. This type of problem has relevance to openwheel racing cars, where local regions of high-speed subsonic flow form under favourable pressure gradients, even though the maximum freestream Mach number is typically considerably less than Mach 0-3. An important consideration for CFD users in this field is addressed in this paper: the freestream Mach number at which flow compressibility significantly affects aerodynamic performance. More broadly, for aerodynamicists, the consequences of this are also considered. Comparisons between incompressible and compressible CFD simulations are used to identify important changes to the flow characteristics caused by density changes, highlighting the inappropriateness of incompressible simulations of ground effect flows for freestream Mach numbers as low as 0.15.

\section{NOMENCLATURE}

C
$C$
$C$
C
h
L

chord

coefficient of lift

coefficient of drag

coefficient of pressure

drag force

minimum height above ground plane

negative lift force (downforec)

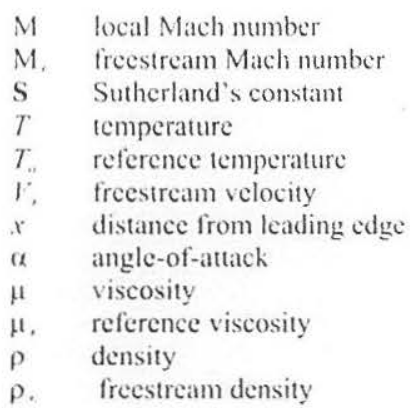

\subsection{INTRODUCTION}

Fluid flow is generally treated as incompressible at Mach numbers up to approximately $0-3$. The incompressible acrodynamics of wings in ground effect have often been studied with regards to applications for vehicles which travel in this range of Mach numbers, such as wing-in-ground-effect (WIG) vehicles and, when the wings are inverted, high-performance racing cars. In the case of the latter, extensive wind tunnel experiments and, more recently, numerical studies have led to a reasonably good understanding of the flow physics. Despite this experience, some areas still require more detailed study, including the influence of compressibility, which has 
Tabie 1

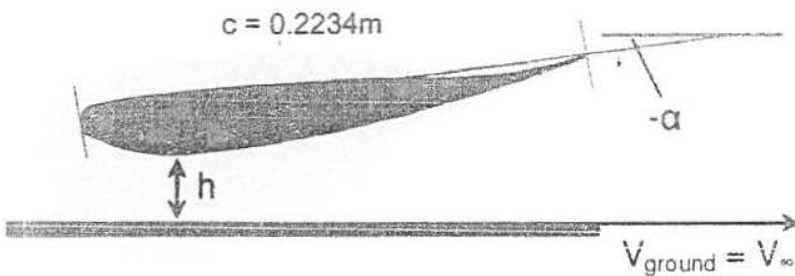

Figure 1. Parameters for the Tyrell 026 inverted aerofoil.
Mach numbers investigated, with equiva!enâ veioctties ant Reynolds numbers

$\begin{array}{lcc}\begin{array}{l}\text { Freestream } \\ \text { Mach number }\end{array} & \begin{array}{c}\text { Equivalent } \\ \text { velocity }\left(\mathrm{ms}^{-1}\right)\end{array} & \begin{array}{c}\text { Regnolds } \\ \text { number }\end{array} \\ 0.088 & 30 & 0.46 \times 10^{\circ} \\ 0.150 & 51.02 & 0.78 \times 10^{\prime \prime} \\ 0.200 & 68.02 & 1.04 \times 10^{\circ} \\ 0.250 & 85.03 & 1.30 \times 10^{\circ} \\ 0.300 & 102.03 & 1.56 \times 10^{\circ} \\ 0.400 & 136.04 & 2.08 \times 10^{\circ}\end{array}$

Given that wings in ground effect, for most applications, tend to be of relatively low aspect ratio and bounded by endplates, a two-dimensional assumption is often made for simple sections ${ }^{(i)}$, although threedimensional flows would exhibit differences. The acroloil was investigated at one angle-of-attack, -3.45 degrees, corresponding io the reference incidence used in other studies of this section ${ }^{-11.1+}$. Four ground clearances were examined $(h / c=1,0-313,0-134$ and 0.067 . where $h$ is measured as the vertical distance from the point on the suction (lower) surface closest to the ground), representative of realistic heights a downforce section would operate at on a typical formula-style vehicle. At each clearance, five freestream Mach numbers. $M_{r}$, from 0.088 (corresponding to Zerihan' $\mathrm{s}^{1+1} 30 \mathrm{~ms}{ }^{1}$ ) to 0.4, were examined. A summary of these conditions is presented in Table 1. The freestream Reynolds number based on chord length was left to increase freely with each increase in frestream Mach number. as it would in real-life for a fixed-chord wing.

A commercial finite-volume Reynolds-averaged Navicr-Stolies solver (Fluent 6.3) was utilised in pressure-based, coupled iniplicit mode, to generate all results presented here. with steady-state cases solved for combinations of the key variables described. Some density-based implicit solutions were computicd for comparison in compressible solutions and found to be near-identical $i<0, i \%$ difference in forces; to those generated by the pressure-based solver over a range of Mach numbers.

Convergence was dictated by stable lift and drag coeflicicnts for each simulation. Compressible cases at the lower two ground clearances and a freestream of Mach 0.4 exhibited strongly unsteady characteristics, and were therefore run as time-dependent solutions with a $0-0002 \mathrm{~s}$ time step. All cases were run in 32-bit double precision using second order node-based upwinding in all discretisation schemes. A standard three-coefficient Sutherland viscosity model:

$\mu=\mu_{0} T / T_{0}{ }^{2}\left(\left(T_{0}+\mathbf{S}\right) /(T+\mathbf{S})\right)$

was used for all cases involving compressible flow, where reference values are; $T_{n}$, reference temperature $=273 \mathrm{~K}, \mu_{i 1}$, reference viscosity $=1.716 \times 10^{\circ} \mathrm{kgm}$ ' $\mathrm{S}^{1}$ and $\mathrm{S}$. the Sutherland constant temperature $=$ $110.56 \mathrm{~K}$. The freestream density was set to $1.225 \mathrm{kgm}^{3}$ in each instance.

Incompressible and compressible (ideal gas) cases were solved for each combination of variables from Table 1, with the yround velocity matching that of the freestream in all simulations in order to accurately reproduce the real-world boundary condition ${ }^{15 s}$. A fitllystructured multi-block grid was generated for each ground clearance. featuring 750 points around the aerofoil and dense regions in the vicinity of the boundary layer (such that the wall $y$ was less than or equal to 1). on the ground, and in the wake. Final grid sizes ranged from approximately 230,000 to 350,000 cells, depending on the ground clearance. Grid convergence tests ${ }^{101}$ were used to establish the most suitable approach, and suggested a $0.2 \%$ difference in the predicted lift force between the grid described and a finer one (up to 500,000 cells); this was deemed an acceptable margin. The domain extended to 20 chord lengths upstream, 18 above and 30 downstream of the aerofoil, based on boundary location tests which showed 


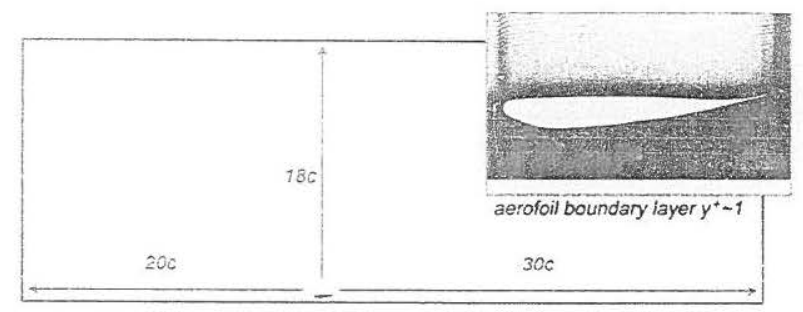

Figure 2. Domain overview, and (insert) mesh detail for an example ground clearance.

negligible influence on the solution at these distances for even the highest Mach number iesied. At low Mach numbers the top boundary could have been placed much closer to the aerofoil and produced some savings in terms of computational effort, but for consistency the one domain size was implemented in all cases. Both this domain and a sample grid are shown in Fig. 2.

Some preliminary work made use of a hybrid mesh, which featured unstructured elements growing from the aerofoil's anisotropic structured ounciary iayer mesi. riowever, inis approaci resulted in occasional inadequate convergence, particularly at the higher mach numbers, when compared to the fully-structured mesh. Therefore this approach was abandoned in favour of the fully-structured mesh.

The realisable $k-(1)$ turbulence model ${ }^{(17)}$ was implemented with an appropriate enhanced wall function based on the method of $\mathrm{Kader}^{1 \mid{ }^{1 \times}}$. This approach was deemed to be effective at best capturing the features of the pressure distribution, ground boundary layer and wake by Mahon ${ }^{(9) 119)}$, whilst yielding trend-accurate force predictions as validated for this study against prior experimental data ${ }^{(7.14)}$. Mahon also notes that the realisable $k-(t)$ model more realistically captures the 'iet' flow which occurs at lower ground clearances ${ }^{(4)}$, whereby the flow velocity does not recover to a freestream value by the trailing edge. This extended region of accelerated flow becomes increasingly important at higher Mach numbers, as will be demonstrated.

With these higher Mach numbers in mind, the realisable $k-(1)$ inodel has also shown good prediction in transonic flows with shock interactions $^{(14)}$, and Gonclaves et $a l^{20}$. note that having a realisable component to the turbulence model is likely to result in markedly more realistic flow features in cases involving transonic buffet, as those at the lowest ground clearances and highest freestream Mach number do in this study. Mentor's $k-\omega$ SST ${ }^{21}$ model has been shown to feature superior prediction in cases featuring buffet $22,2 \geqslant$ and the Spalart-Allmaras model has also been shown effective for a range of transonic flows ${ }^{i * 4}$ including those featuring buffet ${ }^{255_{1}}$. However, given the current lack of experimental data for ground effect situations up to and into the transonic regime, a comparison of turbulence models for these cases would be inconclusive and thus the realisable $k-\varepsilon$ model was retained for the highest-speed simulations presented here.

The flow was assumed to be fully turbulent around the aerofoil, which negates the influence of boundary-layer transition as cxamined in the earlicr low-specd studies on the Tyrell 026 wing $^{(7)}$. Both Zerihan and Mahon ${ }^{(4.11)}$ stipulated a laminar zone in their CFD to account for a transition strip fixed at $10 \%$ of the chord. However, Zerihan reports experimental lift values as much as $20 \%$ lower for the fixed transition case as opposed to the free transition one, which seems excessive even in the context of extreme ground effect. The description of the method of transition discusses the possibility of separation of the flow by the transition roughness strip, and this would have considerable downstream effect on the natural boundary layer separation point as well as immediate lift-generation in the area around the transition strip.

Given this uncertainty and a lack of detailed boundary-layer measurements to validate against, a simple comparison was made in the present CFD between a fully urbulent case and ono which featured a laminar zone to $10 \%$ chord on both upper and lower surfaces. The difference in lift coefficient was found to be within $2 \%$, fairly consistently across a variety of two-equation twrbulence models, at an arbitrarily-chosen $h / c$ of 0.179 at $30 \mathrm{~ms}$. The oneequation model, which is capable of predicting free transition. also placed lift similarly close to the fully turbulent result at this reference velocity.

Witi a laninar zone extending to $30 \%$ of the chord, the predtictict difference increases to ahost $10 \%$, but instabilities which wetht require full unsteady nodelling beconic apparon - indecd, this would appear to be the reason that the experimentalists chose to lix transition at $10 \%$ chord $^{(1+4)}$. At the Reynolds numbers associated with the higher speeds which are the focus of this study, transition would occur earlier than under the experimental concitions at $30 \mathrm{~ms}$ ?. Thus. a fully turbulent simulation is reasonable in this instance.

A sample comparison to the experiments of Zerihan ${ }^{(2)}$ for an $\mathrm{h} / \mathrm{c}$ of 0.179 is shown in Fig. 3; experimental data for higher Wach numbers was not available for the present study. The comparison shows that the numerical approach used for the subsequent $\mathrm{CFD}$. cases was suitable, as it accurately reproduces the $C_{\text {, distribution. At }}$ higher clearances there is a mild over-prediction of the lower surface suction peak, and at the lowest ground clearance, $h / c=0.067$, there exists a more pronounced under-prediction at this point. A conibi nation of factors, in addition to the transition consideration, may have led to this. The experiments were conducted in three dimensions on a finite span wing with endplates, whereas this study is based in two-dimensional modelling, and the large domain of the CFD is not intended to precisely recreate the wind tunnel dimensions. In addition, experimental factors such as the unpredictable nature of the boundary-layer transition and variations in tunnel Reynolds number (between $0.43 \times 10^{\prime \prime}$ and $0.462 \times 10^{\prime \prime}$ based on the aerofoil chord would be amplified at such a very low clearance. Furthermore the uncertainty in height set for the experiments is quoted $^{(1+1)}$ as being around $5-6 \%$ for the 0.067 heighi-to-cherd ravio: enough to make a considerable difference to $C_{p}$ if at the extremes of this range. Overall, the major features of the pressure distributions are well captured, and when compared to predictions by the oneequation model of Spalart and Allmaras and the two-equation $k-(1)$ SST model, the $C_{P}$, results from the realisable model were closest to the experimental values. This result supports the conclusions of Mahon ${ }^{(0,10)}$ with regards to the realisable model.

The compressible solutions obtained at $30 \mathrm{~ms}{ }^{1}$ were near-identical to the incompressible ones for all clearances, with minor differences due to compressibility effects which are discussed in subsequent sections. $C_{L}$ and $C_{D}$ plots are presented in Fig. $4(\mathrm{a})$ and (b). The numerical predictions are offset from the experimental results, due to the fully turbulent nature of the CFD and the other aspects mentioned above. However, the characteristics of the lift and drag slopes are well reproduced, specifically the lift-loss at the lowest ground clearances. The experiments at these lowest ground clearances would have been closest to reproducing true two-dimensional flow at the centrechord position due to the endplates being so close to the ground, and this is where the best correlation with the twodimensional CFD is to be found. Again, these results give confidence that the trends predicted for the present study will be reliable in the absence of validation experiments at the higher Mach numbers.

\subsection{RESULTS}

As one would expect, the incompressible simulations predicted increasing lift and, to a lesser degree, drag, with decreasing ground clearance or increasing Mach number, with corresponding increased aerodynamic efficiency, L/D. Fig. 5(a) and (b) present lift and drag coefficients for the two greater ground clearances, $h / c=1$ and 0.313 . for both incompressible and compressible simulations. It is usefut to 

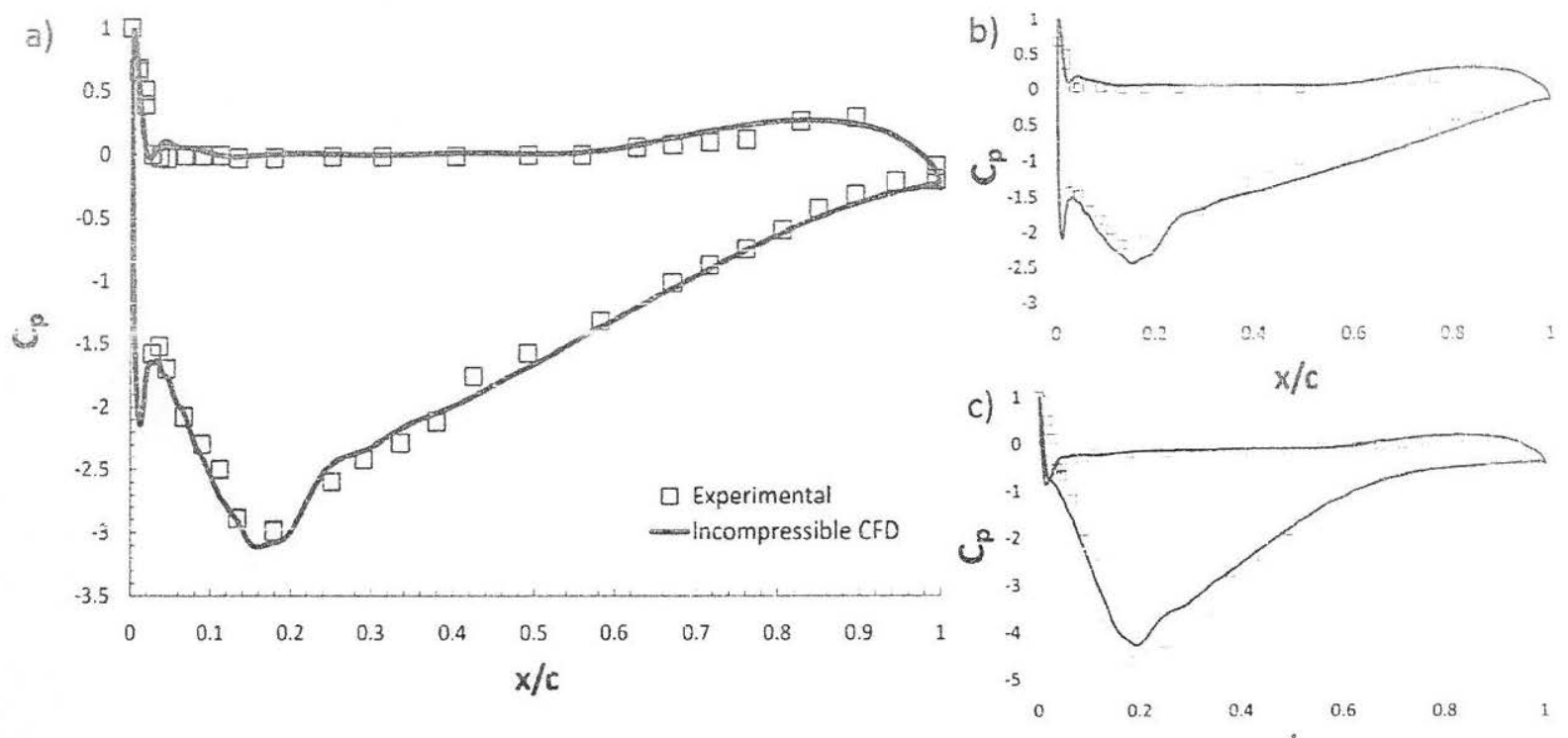

Figure 3. Chordwise pressure coefficient at a) $h / c=0.179$, b) $0.313, c) 0.067$ from CFD, compared to experimental data'

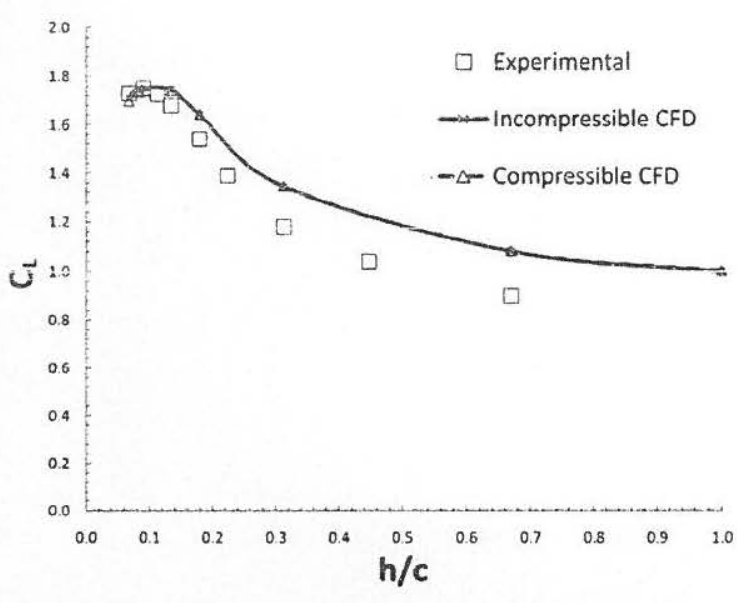

Figure 4(a). Compressible and incompressible lift coefficients from CFD, compared to experimental data for a range of ground clearances ${ }^{(1+1}$.

consider these heights separately from the lower two clearances, due to additional influences at the smaller $h / c$ ratios. If one assumes that the compressible result is the 'correct' one in all cases, then the incompressible result can be viewed as an under or over-prediction, and is expressed as such in terms of percentage in the figures.

Although the difference between compressible and incompressible predictions is negligible at the lowest Mach number, $0-0882$, even then the density around the aerofoil changes by around $1 \%$ of the freestream value in the compressible simulations at these heights. Differences in predicted lift and drag become clearer at Mach 0.15, beyond which trends of increasing disagreement between incompressible and compressible simulations are evident. At this freestream Mach number, the peak Mach number, coincident with the suction peak on the lower surface of the aerofoil, is approximately 0.28 in compressible simulations; $3-4 \%$ higher than the highest incompressible Mach number. The flow in this case is therefore already approaching compressible behaviour, with a density variation around

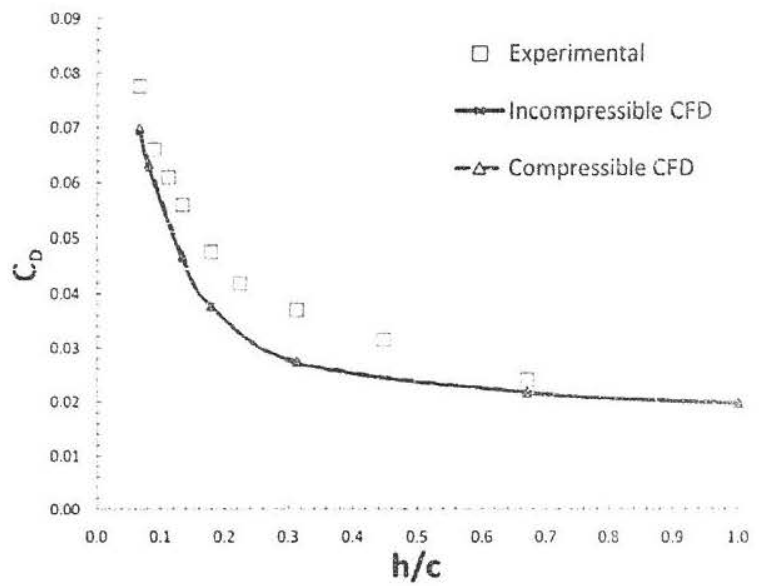

Figure 4(b). Compressible and incompressible drag coefficients from CFD. compared to experimental data for a range of ground clearances $^{(1+1)}$.

the acrofoil of close to $45 \%$ of the freestream at a clearance of $\mathrm{h} / \mathrm{c}=$ 0 313. The increased peak flow velocity induced by the flow compressibility, while yielding a slight decrease in skin friction due to the somewhat thinner boundary layer, also leads to an intensification of the strength and thickness of the wake. It is this effect which is the major contributor to the large relative drag increase in the compressible cases as Mach number rises.

At a freestream Mach number of $0-4$, the peak Mach number is extremely close to sonic in compressible simulations, as compared a peak of $\mathrm{M}=0.74$ in the incompressible case. At $h / c=1$, this results in an underprediction of lift by $-11.5 \%$, and drag $\sim 9 \%$. The increasing constriction of the flow at an $h / c$ of 0.313 leads to the incompressible simulation underpredicting by a far greater margin of $-19 \%$ for $C_{L}$, and $\sim 29 \%$ for $C_{D}$ at the Mach 0.4 freestream.

The simple driving mechanism for the differences exhibited across the board is the density decrease as the flow speeds up around 


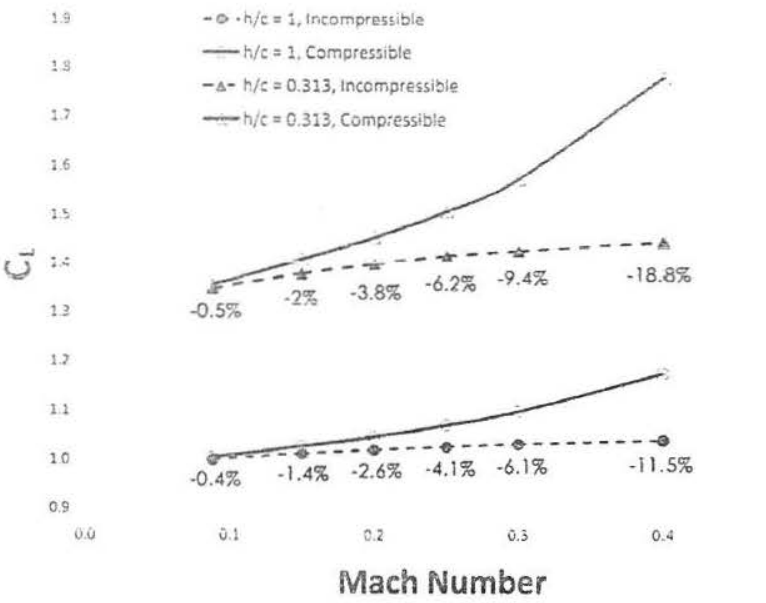

Figure $5(a)$. Compressible and incompressible lift coefficients and percentage incompressible underprediction for $h / c=1$ and 0.313 .

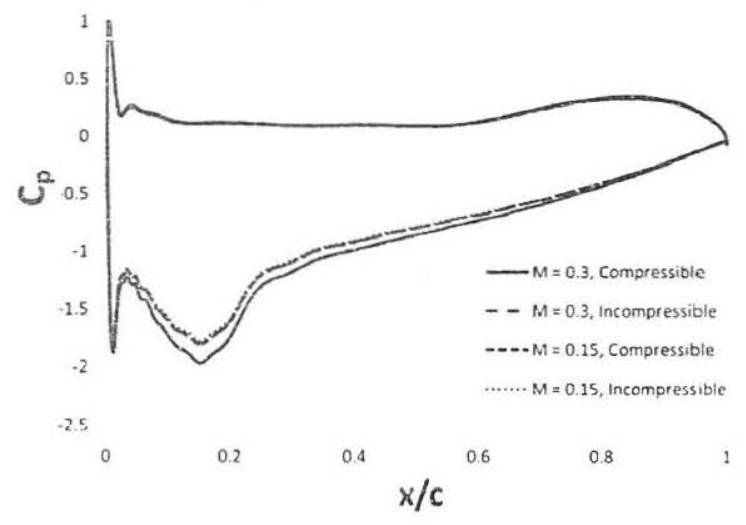

Figure 6(a). Compressible and incompressible pressure coefficients and for $h / c=1, M,=0.15$ and 0.3 .

the suction surface, leading to lower pressure and therefore greater downforce in the compressible cases. As the ground clearance is reduced, the channel through which the air flows narrows, and the effect is exaggerated.

Chordwise pressure coefficients are plotted in Fig. 6(a) and (b) for freestream Mach numbers of 0.15 and 3 to demonstrate the difference between a point at which incompressible and compressible simulations results begin to disagree, and a point at which the incompressible simulation is no longer inappropriate.

One can see from the plots that the higher lift predicted by the compressible simulations is due to a markedly stronger suction peak on the lower surface of the aerofoil, becoming more exaggerated with increasing Mach number and/or decreasing ground clearance. However, the stagnation point and upper surface pressure distribution remains relatively unchanged at both clearances and both Mach numbers. At both $h / c=1$ and $0-313$, the $C_{p}$ calculated by the incompressible solution for a freestream $\mathrm{M}=0.3$ is almost identical to that of the compressible calculation for a freestream $M=0.15$.

Were the trends described here to continue with decreasing ground clearance, one might conclude that a compressible 'correction' could be fairly easily applied to incompressible solutions for this problem, based on fairly minimal additional data reiating to Mach number increases. However, at the two lower

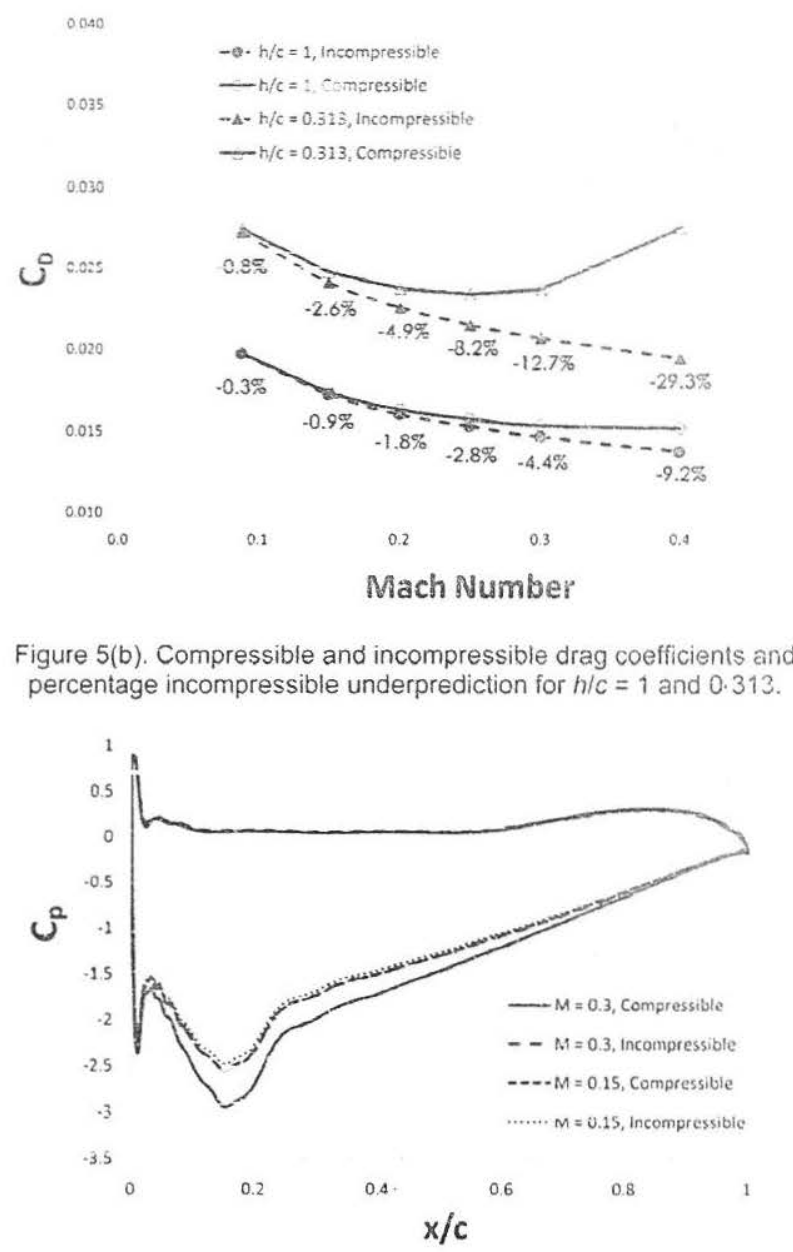

Figure 6(b). Compressible and incompressible pressure coefiicients and for $h / c=0.313, M_{c}=0.15$ and 0.3 .

ground clearances tested, $h / c=0.134$ and 0.067 , these trends give way to much less predictable scenarios. At the lowest $M_{\text {, of }} 0.0882$ for these clearances, the incompressible and compressible solutions already exhibit a non-trivial $\sim 0.7 \%$ difference between each other in terms of predicted lift and drag for both clearances, as density varies by close to $2 \%$ around the aerofoil compared to the freestream value.

Drag follows the same trend as with the two higher clearances as Mach number increases, with incompressible simulations increasingly underpredicting its magnitude. Above a freestream of $\mathrm{M}_{x}=$ 0.15 , the drag coefficient only increases with Mach number in the compressible simulations as seen in Fig. 7(b), while incompressible cases still indicate a decreasing coefficient. Unlike at the two higher clearances, the compressible and incompressible lift coefficients shown in Fig. 7(a) do not differ by much at the two lowest freestream Mach numbers, and indeed the results signal a shift to overprediction by the incompressible simulations.

At $h / c=0.134$, lift .jefficient values for both approaches are within a few percent of each other up to the freestream $M_{c}=0 \cdot 3$. At $h / c=0.067$, the compressible lift coefficient deviates significantly from the incompressible figure at $\mathrm{M}_{\mathrm{x}}=0.15$ onwards, until it begins to decrease at $\mathrm{M}=0.3$ to the extent that the compressible $C_{1}$ is around $15 \%$ lower. If one examines the pressure coefficients presented in Fig. 8(a) and (b), the similarities at $M=0.15$ are under- 


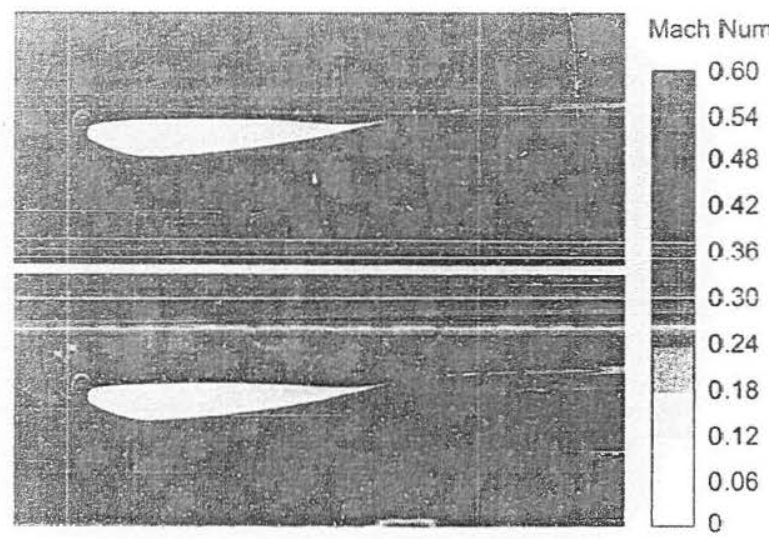

Figure 9(a). Incompressible (top) and compressible (bottom) Mach number contours and for $h / c=0.313, M_{1}=0.3$.

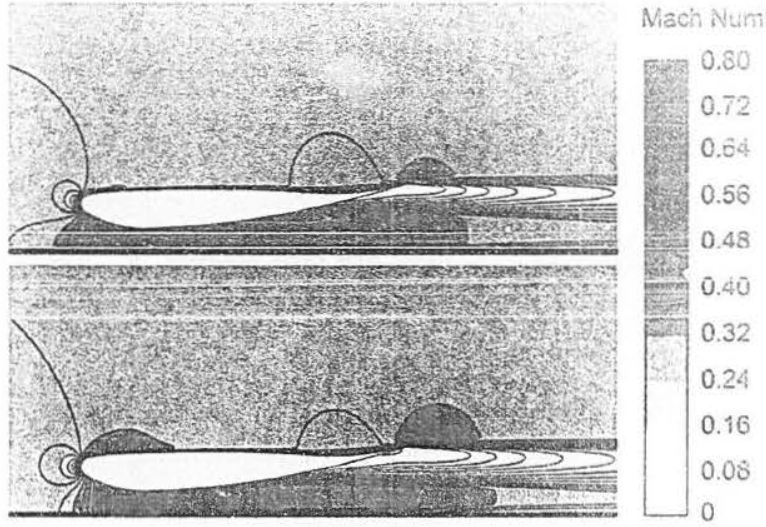

Figure 9(b). Incompressible (top) and compressible (bottom) Mach number contours and for $h / c=0.067, M=0.3$
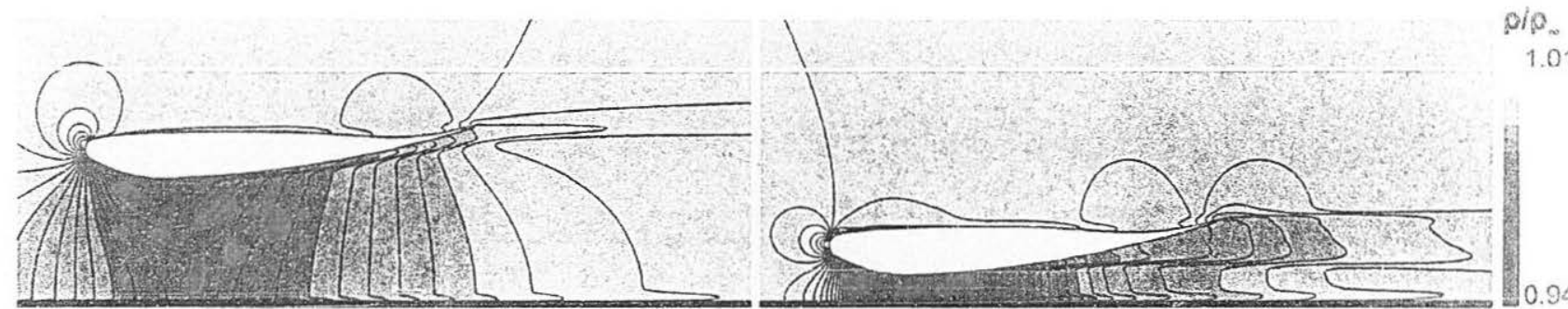

Figure 10. Density variation contours for compressible simulations at $M,=0.15$ ior $h / c=0.313$ and 0.067 .
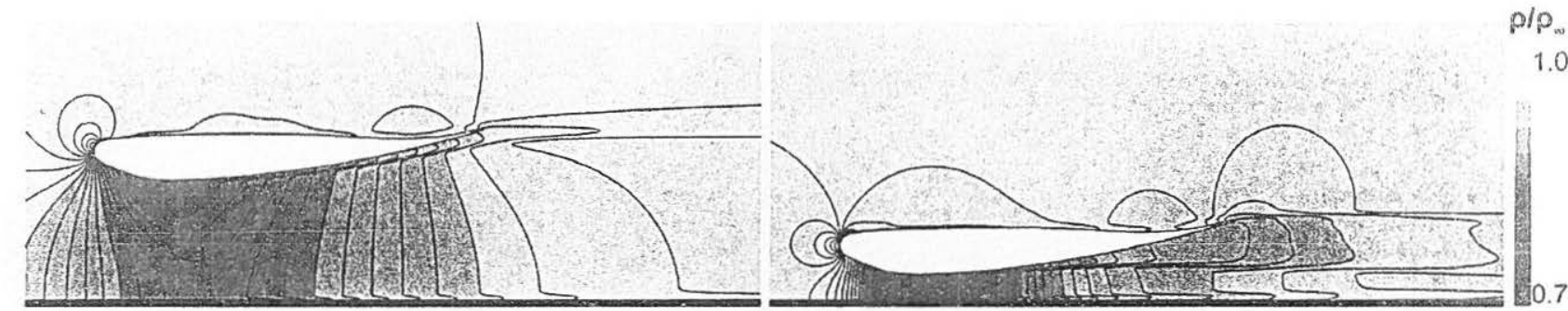

Figure 11. Density variation contours for compressibie simulations at $M_{1}=0.3$ for $h / c=0.313$ and 0.067 .

surface than in its incompressible counterpart, increasing the capacity for lift generation. As the aerofoil is moved closer to the ground, the area ratio between the inlet to the channel and the throat at the point of minimum clearance becomes an important factor. The flow becomes increasingly accelerated and thus the density drops, as can be seen in Fig. 10 and Fig. 11. The volume flow rate therefore increases, yet the mass flow is decreased when compared to the incompressible case, as the stagnation point moves downwards to the suction side, forcing more flow over the upper surface. This results in an effective change of camber, and thus while the peak suction is greater, the overall lift generated over the fore section of the acrofoil is less, and the gradient is more pronounced to the trailing edge, as can be seen referring back to Fig. $8($ b). The resultant thicker, stronger wake makes up the majority of the extra drag when compared to the incompressible case, and these effects are further exaggerated with increasing Mach number.
In addition, more so than at $h i c=0.134$, the wake at the clearance of $h / c=0.067$ deflects noticeably towards the ground, as evidenced in Fig. 9(b), to the extent that it begins to interact signiticantly with the ground boundary layer at around two chord lengths downstream of the trailing edge. This is due to the highly separated flow effectively changing the camber of the acrofoil again, deflecting the wake downwards in a similar manner as would be achieved with a downwards deflection of a trailing edge flap.

As mentioned previously, at $\mathrm{M}_{\mathrm{x}}=0.4$ for the two lower ground clearances, a normal shock wave forms between the aerofoil and the ground in compressible simulations. The lift and drag coefficients presented in Fig. 7(a) and (b) for this Mach number are timeaveraged from unsteady simulations, and while they exhibit a highmagnitude difference in lift and drag when compared to the incompressible values (at $h / c=0-067, C_{L}$ is $\sim 35 \%$ less and $C_{D}$ is over $100 \%$ greater), the values themselves do not tell the full story. 

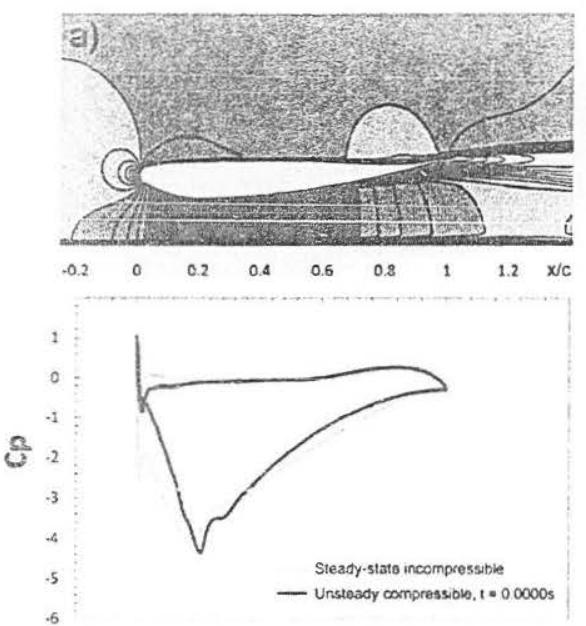

(d)
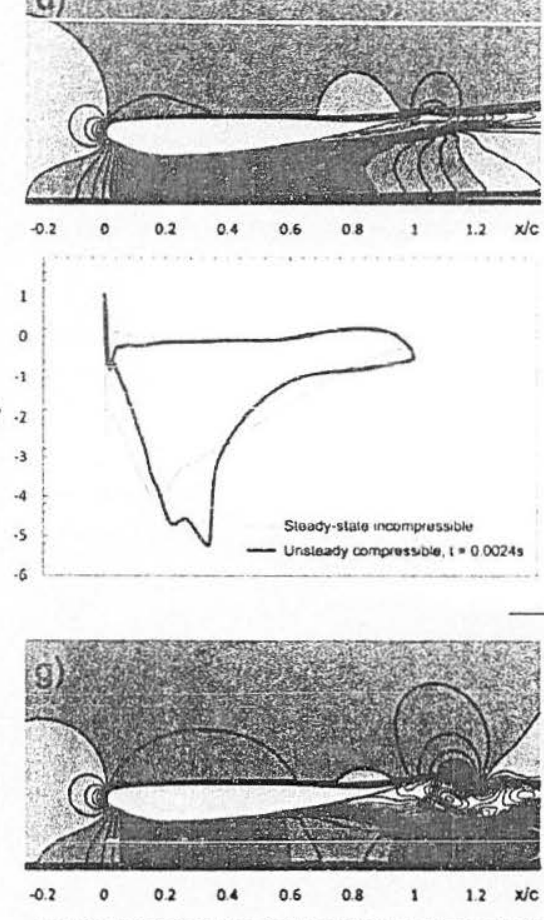

1
0
8
8
8
-3
4
5
-6

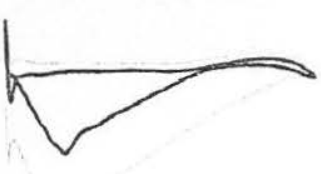

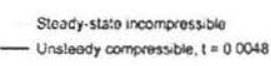

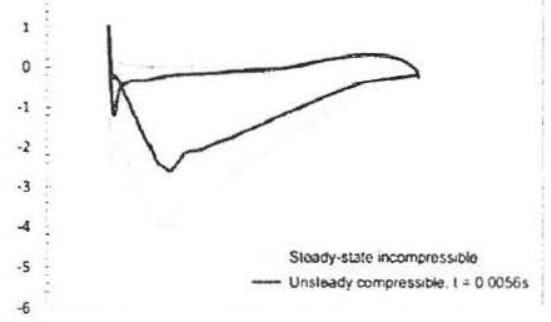

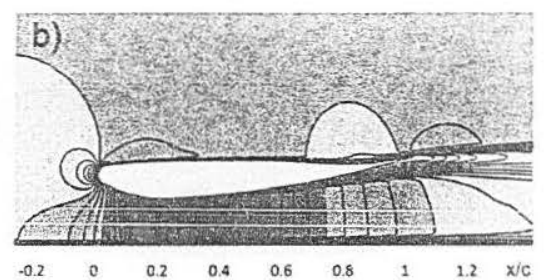

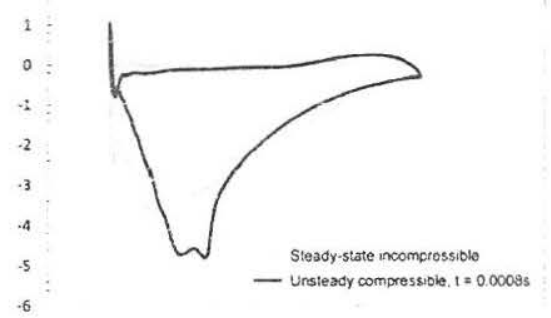

$-6$
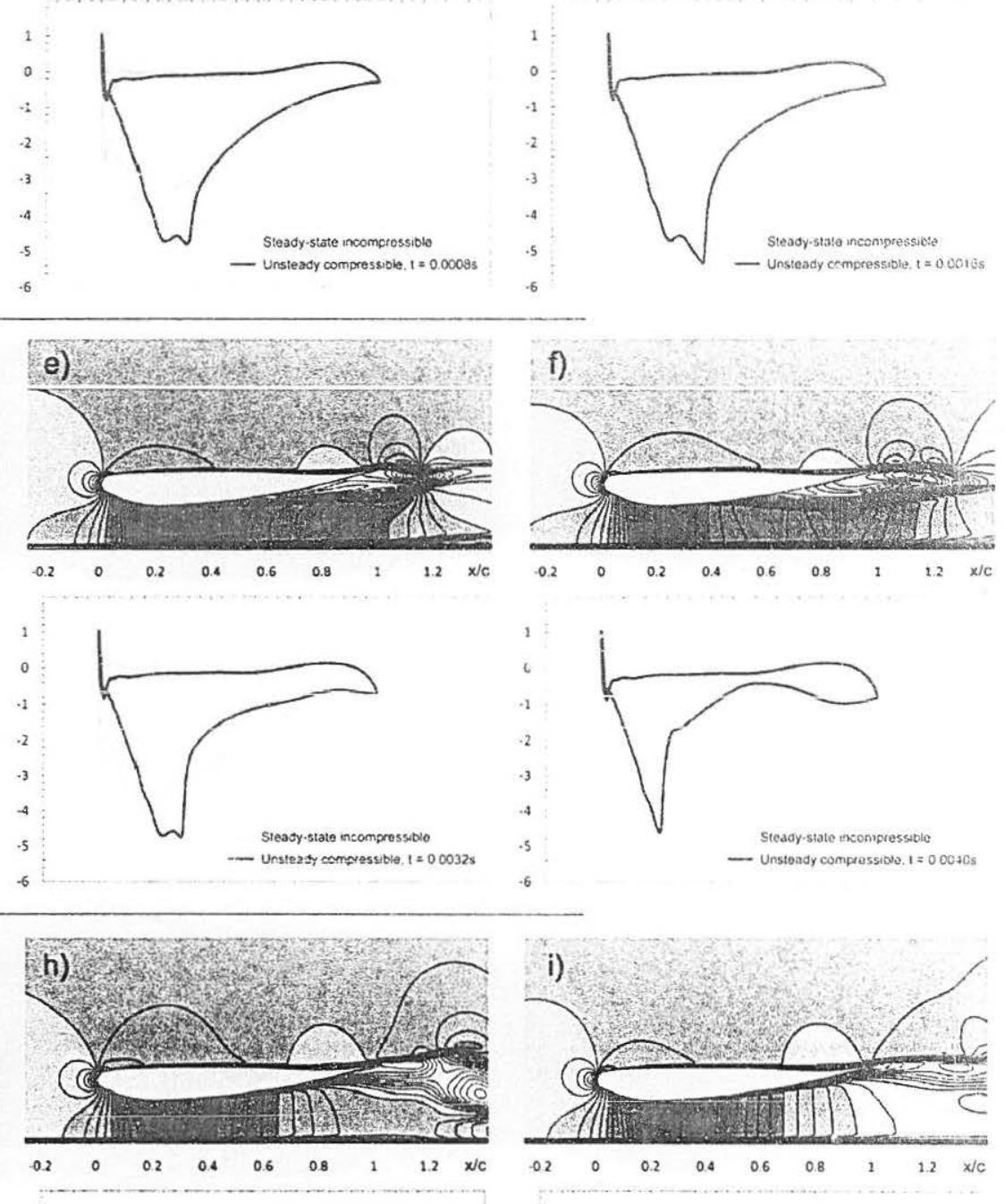

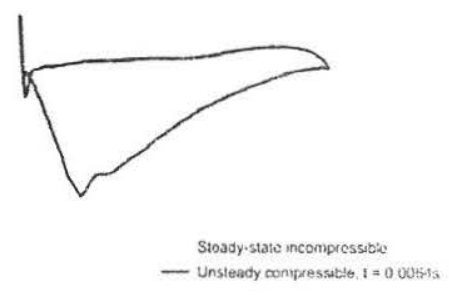

Figure 12. Mach number contours and coincident chordwise pressure coefficient plots depicting one complete buffet cycle, at $h / c=0 \cdot 134$ and $M_{x}=0.4 . t=0.000$ represents the start of the cycle, which was in this instance the third in the simulation. 
Figure 12 presents Mach number contours and coincident pressure coefficient plots for the unsteady case at $h / c=0.134$. As the flow exhibits a definite and consistent period of natural shock movement and shear layer shedding (self-sustained transonic buffet), this series of images presents the behaviour over one complete cycle (a realtime of approximately 0.0064 seconds) of the $156.25 \mathrm{~Hz}$ oscillation. Instantaneous pressure coefficient plots at regular time-steps through the transient period are compared to the steady-state incompressible result for reference.

initiaily, (Fig. 12(a)), the fiow becomes marginaliy supersonic at the narrowest point of the channel created by the aerofoil and the ground while the boundary layer is separated towards the rear of the suction surface as a remnant of the previous cycle. The reduced density means that the local speed of sound is as low as $310 \mathrm{~ms}^{-1}$ and the compression waves which are starting to coalesce have, by $t=0-0008 \mathrm{~s}$, formed a strong normal shock wave in the channel. The shock wave propagates downstream over the coming time steps with the maximum local Mach number greater than 1.3 in Figs 12(c), (d) and (e). The shock reaches its downstream limit iocation of approximately $0.4 x / c$ in Fig. 12 (d), after 0.0024 seconds. The boundary layer is separated by the foot of the shock on the acrofoil surface, and therefore as the suction peak reaches its maximum, the adverse pressure gradient over the rearward portion of the lower surface grows more significant and the amount of downforce generated begins to sur̂ter.

The large-scale separation results in a shear layer which, between $t=0.0040 \mathrm{~s}$ and $0.0048 \mathrm{~s}$, has effectively created a second throat. This re-accelerates the flow to close to supersonic again (indeed, at a slightly higher freestream Mach number, it does locally exceed Mach 1 for a second time). As the shock wave recedes towards the leading edge in Fig. 12(e) and (f), it re-enters the region of lower upstream velocities, and weakenis until the flow returns to a fully subsonic, shockless state by Fig. 12(g).

The aerofoil's capacity for lift generation is detrimentally affected during the shoch's upstream movement. However, the eventual absence of the shock in the channel removes the driver for the boundary layer separation, and thus a period of re-attachment and lift-recovery is initiated that will eventually lead to the shock wave forming again and the process repeating. These phenomenological observations are consistent with the theories of $\operatorname{Lec}^{(2 n-2 N)}$, in which the movement of the shock creates pressure waves which travel downstream through the separated flow region to the trailing edge. whereupon an upstream-travelling wave which eventually interacts with the shock and imparts the energy required to move it upstream. Such behaviour has been observed computationally with unsteady RANS in other studies ${ }^{(23.25)}$ and thus the observed flow behaviour seems plausible albeit, at this stage, not validated experimentally. The cyclical variations in lift and drag are plotted in Fig. 13, and the $156.25 \mathrm{~Hz}$ frequency seems reasonable when compared to existing buffet studies on less cambered aerofoils ${ }^{(23-2 x)}$.

Several other interesting flow features present themselves during this cycle: the ground boundary layer appears to also be separated by the shock wave and forms a significant bubble moving with the ground downstream of the trailing edge as the shear layer is shed (not fully pictured in Fig. 12). One can also see that the stagnation point position fluctuates around the leading edge as the flow under the aerofoil reacts to the rhythmic formation and dissolution of the shock wave.

At $h / c=0.067$ ground clearance, similar flow behaviour is observed albeit with an even greater difference between maximum and minimum lift and drag generation during the period, which occurs at almost exactly the same frequency. It is worth noting that the critical freestream Mach number at this height is approximately 0.35 , with transonic buffet cycles immediately accompanying the appearance of supersonic flow.

Naturally, such intense buffet behaviour would occur suddenly and have ramifications not only as discussed for the aerodynamic performance of the aerofoil, but structural problems would become a major concern due to high-frequency vibrations and rapid changes in pitching moment.

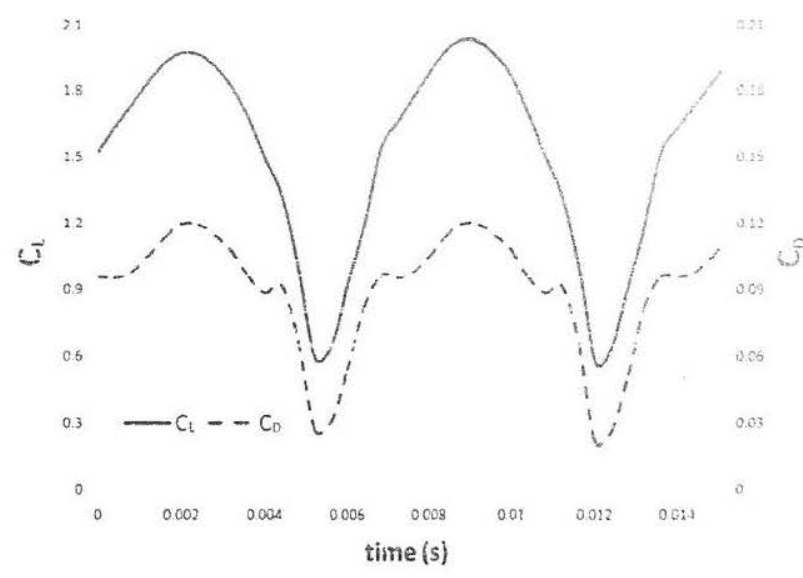

Figure 13. Variation in lift and drag coefficients with time for case depicted in Fig. 12.

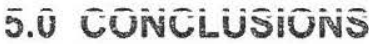

CFD has been successfully used to determine the effects of the onset of compressibility for a two-dimensional aerofoil in ground effect, by examining the differences between incompressible and compressible simulations for a range of ground clearances, and freestream Mach numbers up to 0.4 . The main conclusions can be summarised as follows:

- Due to the highly accelerated flow between the acrofoil and the ground, compressibility effects alter the flow to produce different lift and drag forces compared to those which would he obtained from incompressible simulations, even at a relatively low freestream Mach number of 0.15. These effects become more significant as Mach number is increased.

- At higher ground clearances of $h / c=1$ and 0.313 , lift and drag forces are increasingly underpredicted by incompressible simulations as Mach number is incremented, due to the lower density region between the suction surface and the ground accelerating the flow and producing both a stronger suction peak and wake.

- At lower ground clearances of $h / c=0.134$ and 0.067 , the trends in lift are towards overprediction by incompressible simulations, as the more accelerated compressible flow produces low pressure over a much shorter region to satisfy conservation of momentum. Separation occurs earlier, markedly thickening the wake.

- At the two lower clearances the critical freestream Mach number exists between 0.35 and 0.4 . Reaching this point results in highly unsteady transonic buffet flow, featuring periodic formation of a shock wave and large-scale separation. This has a considerable detrimental effect on the production of lift, and drag is substantially increased.

Clearly, simple compressible corrections could not be successfully applied to incompressible solutions given the sensitivity of the flow to ground clearance. Although compressibility influences on forces were the main focus of this study, the changes to the pressure distributions as compared to incompressible results would have additional consequences for the predicted aerofoil pitch characteristics and any downstream components affected by the altered wake.

If one were to extend the consideration of compressible effects to, for instance, a full open-wheel racing car. density changes in the flow would likely be important for the car underside and diffuser. Another important region for compressibility is in the contact patch between tyres and the ground, where the ait is forced into a narrow space causing pressure coefficients that exceed unity ${ }^{(2 \%)}$. 
The critical freestream Mach number at the lowest ground clearance is approximately Mach 0.35 , which equates to $\sim 119 \mathrm{~ms}$ (or close to $266 \mathrm{mph}, 428 \mathrm{kmph}$ ). While this is somewhat higher than a current Formula One or Indy-style racing car, the speed is within the range of less conventional vehicles which may feature downforce wing sections, such as top-fuel dragsters or land speed record cars. Indeed, a modified Honda Fl car reconfigured as a land speed record contender, the 'Bonneville 400 ' car, achieved around Mach $0 . \overline{3} \bar{z}$ $(400 \mathrm{~km} / \mathrm{h})$ at ambient conditions ith a low-drag front wing fitted. The occurrence of a shock wave would have a considerable destabilising effect for such vehicles.

Work is currently underway to continue this study with a threedimensional wing to examine the compressibility effects described here in greater and more realistic detail, including a programmc of experiments which will provide necessary data for CFD validation and further exploration of the flowfield features which incompressible simulations would not predict.

\section{REFERENCES}

1. Zhang, X., TOET, W. and Zerhan, J. Ground effect aerodynamics of race cars, Applied Mech Reviews, 2006, 59. pp 33-49.

2. Kim, C.S., ChOngam, K. and Rho, O.H. Computations of high-lift aerofoil flows using two-equation turbulence models AIAA 99-0542.

3. KATZ, J. Aerodynamics of race cars, Anmual Review of Fluid Mech, 2006, 38, pp 27-63.

4. KATZ, J., Calculation of the aerodynamic forces on automotive lifting surfaces, ASME J Fluids Eng, 1985, 107, pp 438-443.

5. Ranzenbach. R. and BARLOW, J. Two-dimensional acrofoil in ground effect, an experimentai and computational study. 1994. SAE Paper 94. 2509.

6. RANZENBACH, R. and BARLOW, J. Cambered aerofoil in ground effect: an experimental and computational study, 1996. SAE Paper 96-0909.

7. ZERIHAN, $J$. and ZHANG, $X$. Acrodynamics of a single clement wing in ground effect. $J$ Aircr. 2000, 37, (6), pp 1058-1064.

8. ZERIHAN, J. and ZHANG. X. Aerodynamics of a double element wing in ground effect, ALAA J, 2003, 41, (6), pp 1007-1016.

9. MAHON, S. and ZHANG, Z. Computational analysis of pressure and wake characteristics of an aerofoil in ground effect, J Fluids Eng. 2005. 127. pp 290-298.

10. MAHON, S and Zhang, Z Computational analysis of a inverted doubleelement aerofoil in ground effect. $J$ Fluids Eng, 2006. 128. pp 1172 1180.

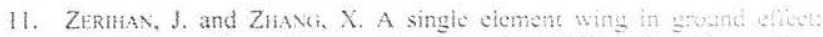
comparisons of experiments and computation, 2004, AiAd Pater 20010423 .

12. Roller. S, and MilN. C.D. A low Mach number selieme bascd on? multi-scale asymptotics. Computing and thualizution in Sitemet. 2000 , 3. (1/2). pp 85-91.

13. Keshtibax, I.J. Bel.berda. F. and Webstir. M.F. Compressithic fiow solvers for low Mach number flows - a review. 2004. University at Waies Computer Science Report CSR-2004.

14. ZERIHAN, J. An Investigation into the Acrodynamics of Wings in Grotnd Effect. 2001. PhD thesis. University of Southampton. Sehed of Engineering Sciences.

15. Barber T.J., LeONARDI E. and ARCher R.D. Causes for Jiscrepancies in ground effect analyses, Aeronaut $J$. 2002. 106. (1066), pp 653-657.

16. ROACHE, P.J. Quantification of uncertainty in computational fluid dynamics, Annual Review of Fluid Mech, 1997, 29. pp 123- 660.

17. Shih, T.H., Liov, W.W. Shabir. A.. Yaydi, Z. and Zill, J. A new k-10 eddy-viscosity model for high Reynolds number turbulent flows model development and validation. Computers of Flutits, $1995,24.13)$. pp 227-238

18. B. KADER. Temperature and concentration profiles in fully turimient boundary layers. Im J Heat Mass Trinsfer, 1981. 24. (9). pp 1541-154-3.

19. Liov. W.W., Huavg, G. and Shin. T.H. Turbulence model assessment for shock wave/turbulent boundary-layer interaction in tansonic and supersonic flows, Computers and Fluits, 2000, 29. pp 275-299.

20. GonClaves, E. and Hoedevilet. R. Turbulence mode! and numerical scheme assessment for buffet computations. Int J for Nimerical Methods in Fluids, 2004, 46, pp 1127-1152.

21. Mester. F.R. Two-equation eddy-viscosity iurbulence models for engincering applications, A/A.4 J. 1994, 32. (\$), pp $1598-1605$.

22. Spalart, P. and Al.lmaras. S. A ene-equation uirbulence nodel fir aerodynamic flows. 1992. AlAA Paper 92-(0439.

23. Brunet, V. Computational study of butfet phenomenon with unstezity RANS equations. 2003. 21st AlAA Applied Aerodynamics Conierenee. AIAA Paper 2003-3679.

24. XlaO, Q. TSAi, H.M. and LIt, F. Numerical study of transonic buffet on a supercitical aerofoil. Al.4. J. 2006. 44. (3). pp 62()- 628.

25. Dicix. S. Numicrical simulation of transontic buffet over a supereritical atrotoi! . 4144 J, 2005, 43. (7), pp !556-1566.

26. LiE, B.H.K. Transonic butfet on a supereritical acrofoil. Aetomatal $f$ 1990, 94, (935), pp 143-152.

27. LEE. B.H.K. Oscillation shock motion caused by transonic shock boundary layer interaction. A/A.t J. 1990, 28. (5), pp (9) +2-9)+4.

28. LEE, B.H.K. Self-sustained shock oscillations on aerofoils at transonic speeds. Prog in derospace Sci. 2001, 37, pp 147-196.

29. FACKREI.L. J.E. The Acrodynamics of an isolated Wheet Rotating in Contact with the Ground. 1974. PhD thesis, University of Lomden. 